\title{
Treating BRAFV600E metastatic colorectal patients in 2019: a BEACON of hope?
}

\author{
Christelle de la Fouchardiere
}

Department of Medical Oncology, Centre Léon Bérard, Lyon, France

Correspondence to: Christelle de la Fouchardiere. Department of Medical Oncology, Centre Léon Bérard, 28 rue Laennec, 69008 Lyon, France.

Email: christelle.delafouchardiere@lyon.unicancer.fr.

Provenance: This is an invited article commissioned by the Editorial Office, Annals of Translational Medicine.

Comment on: Van Cutsem E, Huijberts S, Grothey A, et al. Binimetinib, Encorafenib, and Cetuximab Triplet Therapy for Patients With BRAF< V600E-Mutant Metastatic Colorectal Cancer: Safety Lead-In Results From the Phase III BEACON Colorectal Cancer Study. J Clin Oncol 2019;37:1460-9.

Submitted Aug 19, 2019. Accepted for publication Aug 28, 2019.

doi: 10.21037/atm.2019.08.110

View this article at: http://dx.doi.org/10.21037/atm.2019.08.110

BRAF V600E mutations have been described, since their discovery date in 2002 , in about $10 \%$ of metastatic colorectal (mCRC) patients (pts) included in prospective clinical trials or retrospective studies (1-3). However, this rate could have been underestimated as suggested in a prospective population-based cohort and confronted by clinical observations $(4,5)$. Indeed, whereas RAS testing is recommended since 2009 in first-line mCRC treatment, the recommendations for BRAF V600E mutations testing have only been updated recently, leading to a significant number of patients lacking this information in the first-line metastatic setting (6). Furthermore, BRAF V600E mCRC pts are often older, with poorer performance status than BRAF wild-type pts with non-measurable nodal and/or peritoneal metastases, excluding them from clinical trials (7). At last, their prognosis being poor, BRAF mutant mCRC pts may die without receiving any specific treatment, sometimes even before any referral in oncology (5). However, significant progresses have been made in the last 5 years concerning BRAF V600E mCRC pts and major information have been highlighted: First, the use of the folfoxiri-bevacizumab combination has emerged as standard first-line chemotherapy following the results of the TRIBE study $(3,8)$. In spite of the low number of BRAF V600E mCRC pts $(n=28$ pts including 16 treated with folfoxiri bevacizumab and 12 treated with folfiri-bevacizumab) included in this study, the response rate of $56 \%$, median progression-free-survival (mPFS) of 7.5 months and median overall survival (mOS) of 19.0 months were impressive enough to be accepted by all experts as a "standard first-line therapy" for BRAF V600E mCRC pts. Conversely, the benefit of adding an anti-EGFR to chemotherapy in the first-line metastatic setting in BRAF V600E mutant mCRC is still controversial (9-11) even if a doubt has emerged from the results of a randomized phase II study showing a surprisingly high response rate $(64.7 \%)$ in BRAF V600E pts with the combination of panitumumab to folfoxiri (versus folfoxiri alone) but without any impact on mPFS (12). Most of the guidelines currently recommend triplet chemotherapy-bevacizumab as the first-line treatment if the patient fits for it. In case of poor general status or advancedage, a doublet of chemotherapy plus bevacizumab is another option. Knowing the association between BRAF V600E mutations and mismatch repair deficiency (dMMR) and following the recent evidences demonstrating that $\mathrm{d} M M R$ is a predictive biomarker for immunotherapy, it emphasized the need for dMMR testing in BRAF V600E mCRC (13-16). Indeed, it has been shown that pts with BRAF V600E and microsatellite instability (MSI) tumors have the same benefit of immune-checkpoint inhibitors than BRAF wild-type pts (15). Additionally, considering the aggressiveness of BRAF V600E mCRC, it has been questioned by several teams if the resection of liver metastases could be useful $(17,18)$. The results of retrospective studies confirmed the poor-prognosis effect of BRAF V600E mutations even after curative-intent surgery with disease-free-survival and overall-survival poorer than in BRAF wild-type pts but agreed on the statement that 
long-term survival was possible. Then, BRAF V600E mCRC pts should not be excluded from surgical strategies if feasible. Finally, BRAF-specific inhibitors (such as vemurafenib, dabrafenib and encorafenib etc.) have been evaluated since many years in BRAF V600E mCRC, alone or in combination with EGFR and MEK inhibitor (19-21). Some interesting results have been presented in a randomized phase II study with the combination of vemurafenib to irinotecan and cetuximab (22). However, the greatest hope comes from another triple combination against BRAF V600E mutant mCRC. The first results of the BEACON study, a randomized phase III study, were recently published in the Fournal of Clinical Oncology by Van Cutsem $e t$ al., the authors reporting the safety lead-in part of this clinical trial (23). The BEACON study was an openlabel, randomized, three-arm, phase III study evaluating in patients with BRAF V600E-mutant mCRC the efficacy and safety of the combination of encorafenib, a third-generation BRAF inhibitor, plus cetuximab with or without binimetinib, a MEK inhibitor. The control arm was the investigators' choice, based on cetuximab, irinotecan +/fluorouracil and folinic acid. The randomisation was in a 1:1:1 ratio. Patients were required to have metastatic BRAF V600E-mutant mCRC, progressive after one or two prior regimens. The determination of the BRAF status was local but centrally confirmed. All other inclusion criteria were classical, particularly for 0-1 ECOG-PS (Eastern Cooperative Oncology Group Performance Status) and RECIST (Response Evaluation Criteria in Solid Tumors) version 1.1 criteria. As the BEACON study was designed without having any safety results for the triplet combination (encorafenib, cetuximab and binimetinib), it was decided to conduct a 30-patients Safety Lead-In (SLI) analysis. The SLI was carried out in 7 sites selected in 4 countries (Belgium, Netherlands, Spain, and USA). The primary end point was the triplet combination safety analysis, including dose limiting toxicities (DLTs), incidence and severity of adverse events (AEs) and incidence of dose interruptions, modifications, and discontinuations. But authors reported also treatment's efficacy, evaluated by confirmed overall response rate (ORR), duration of response (DOR), progression-free survival (PFS), time to response, and overall survival (OS). Tumor assessments were performed every 6 weeks for the first 24 weeks, then every 12 weeks until disease progression, withdrawal of consent, or initiation of subsequent anticancer therapy. Patients were mainly included after only 1 line of prior chemotherapy $(60 \%)$. Their characteristics were as expected for BRAF- mutant mCRC pts, a majority of them harboring right-side colon tumor, frequent nodal (50\%) and peritoneal (37\%) metastasis. On the contrary, the median age was unexpectedly young for this pts population (59 years; range, 38-77), especially since few pts had a MSI-H tumor $(\mathrm{n}=1 ; 3 \%)$. The triplet combination led to DLTs in 5 pts out of 30, including 2 serous retinopathy, 1 reversible decreased left ventricular ejection fraction, both related to the MEK inhibition, and 2 cetuximab-related infusion reactions. The most frequently reported all-grade adverse effects (AE) were gastrointestinal including diarrhea (76.7\%), nausea (63.3\%) and vomiting (50\%). Dermatological AE were also frequently described [acneiform dermatitis (66.7\%), dry skin $(50 \%)]$. Fatigue and decreased appetite were reported in more than half of the pts. Grade 3-4 toxicities were reported in 21 patients $(70 \%)$, the most frequent being fatigue (13\%; all grade 3$)$ and urinary tract infections $(10 \%$; all grade 3$)$. The most common $(\geq 10 \%)$ grade 3-4 laboratory abnormalities were anemia, AST increasing and creatine phosphokinase increasing. AEs led to $6(20 \%)$ study drug discontinuation. Because one pt was found to have a non-V600E BRAF-mutant mCRC, efficacy analysis was analyzed in 29 pts. The ORR per local assessment was $48 \%$ (95\% CI, $29.4 \%$ to $67.5 \%$ ) and $41 \%$ (95\% CI, $23.5 \%$ to $61.1 \%$ ) by retrospective central assessment. Two pts presented a centrally-confirmed complete tumor response (7\%) and 10 a partial response (34\%). The triplet combination resulted in an early reduction in tumor size with $75.0 \%$ of responding patients achieving response within 2 months but it was also prolonged with a median DOR of 8.1 months (95\% CI, 2.8-NR) according to the central assessment. With a median follow-up time of 18.2 months (16.6-19.8 months), median PFS (mPFS) was 5.5 months (95\% CI, 4.2-9.3 months) per central assessment and mOS was 15.3 months (95\% CI, 9.6-NR). The 12 -month OS rate was $62 \%$ (95\% CI, 42.1-76.9\%). Locally-assessed mPFS was similar for patients who received either one or two prior regimens: 8.0 (95\% CI, 5.6-9.7) and 7.7 (95\% CI, 4.1-10.8) months respectively.

The first results of this safety-lead-in part of the phase III BEACON clinical trial showed that the triplet combining encorafenib + cetuximab + binimetinib was tolerable and efficient in the second-line metastatic setting for BRAF V600E mCRC pts even if the number of pts is low to reach a solid conclusion. The main toxicity was related to MEK inhibition with well-known retinal AEs and risk of cardiac insufficiency. Furthermore, the DLTs have also included 2 cetuximab-related infusion reactions 
without any DLT due to the BRAF inhibitor, encorafenib. The efficiency was promising in the 29 treated pt, especially in terms of early and durable tumor responses. This may be interesting in BRAF V600E mCRC pts likely to experience pain or other complications because of the tumor burden (due to retroperitoneal nodes or peritoneal carcinomatosis in particular). It will be interesting to see if early tumor reduction at 8 weeks is significantly correlated with PFS and $O S$ as it has been demonstrated for antiEGFR in RAS wild type mCRC (24). Furthermore, quality-of-life assessments should also provide additional context to see the benefit of reducing the tumor burden. The first results of the BEACON study concerning the entire population were presented at the ESMO-GI meeting in July 2019 confirming the superiority of the triplet combination over the investigator's choice arm in terms of response rate and mPFS (25). We can certainly affirm that this study provides a beacon of hope for pts with BRAF V600E pts. Still, there are unanswered questions concerning the added value of the MEK inhibitor to the doublet combination, the efficacy of the combination in MSI BRAF V600E mCRC pts, the number of resected pts and their outcome etc. Moreover, if the efficiency results of the triplet anti BRAF combination is confirmed as better in less heavily pretreated pts, the phase II study ANCHOR (NCT03693170), currently evaluating the triplet in first line metastatic setting, will bring us interesting results.

\section{Acknowledgments}

None.

\section{Footnote}

Conflicts of Interest: C de la Fouchardiere declared providing a consultancy role for Pierre Fabre, Roche, Amgen, Bayer, Lilly, BMS and Servier. C de la Fouchardiere has been involved as investigator in the BEACON study.

Ethical Statement: The author is accountable for all aspects of the work in ensuring that questions related to the accuracy or integrity of any part of the work are appropriately investigated and resolved.

\section{References}

1. Venderbosch S, Nagtegaal ID, Maughan TS, et al. Mismatch repair status and BRAF mutation status in metastatic colorectal cancer patients: a pooled analysis of the CAIRO, CAIRO2, COIN, and FOCUS studies. Clin Cancer Res 2014;20:5322-30.

2. de la Fouchardière C, Cohen R, Malka D, et al. Characteristics of BRAF V600E Mutant, Deficient Mismatch Repair/Proficient Mismatch Repair, Metastatic Colorectal Cancer: A Multicenter Series of 287 Patients. Oncologist 2019. [Epub ahead of print].

3. Cremolini C, Loupakis F, Antoniotti C, et al. FOLFOXIRI plus bevacizumab versus FOLFIRI plus bevacizumab as first-line treatment of patients with metastatic colorectal cancer: updated overall survival and molecular subgroup analyses of the open-label, phase 3 TRIBE study. Lancet Oncol 2015;16:1306-15.

4. Sorbye H, Dragomir A, Sundström M, et al. High BRAF Mutation Frequency and Marked Survival Differences in Subgroups According to KRAS/BRAF Mutation Status and Tumor Tissue Availability in a Prospective PopulationBased Metastatic Colorectal Cancer Cohort. PLoS One 2015;10:e0131046.

5. Chu JE, Johnson B, Morris VK, et al. Population-based screening for BRAF V600E in metastatic colorectal cancer (mCRC) to reveal true prognosis. J Clin Oncol 2019;37:3579.

6. Benson AB, Venook AP, Al-Hawary MM, et al. NCCN Guidelines Insights: Colon Cancer, Version 2.2018. J Natl Compr Canc Netw 2018;16:359-69.

7. Clancy C, Burke JP, Kalady MF, et al. BRAF mutation is associated with distinct clinicopathological characteristics in colorectal cancer: a systematic review and meta-analysis. Colorectal Dis 2013;15:e711-8.

8. Loupakis F, Cremolini C, Salvatore L, et al. FOLFOXIRI plus bevacizumab as first-line treatment in BRAF mutant metastatic colorectal cancer. Eur J Cancer 2014;50:57-63.

9. Di Nicolantonio F, Martini M, Molinari F, et al. Wildtype BRAF is required for response to panitumumab or cetuximab in metastatic colorectal cancer. J Clin Oncol 2008;26:5705-12.

10. Pietrantonio F, Petrelli F, Coinu A, et al. Predictive role of BRAF mutations in patients with advanced colorectal cancer receiving cetuximab and panitumumab: a metaanalysis. Eur J Cancer 2015;51:587-94.

11. Stintzing S, Miller-Phillips L, Modest DP, et al. Impact of BRAF and RAS mutations on first-line efficacy of FOLFIRI plus cetuximab versus FOLFIRI plus bevacizumab: analysis of the FIRE-3 (AIO KRK-0306) study. Eur J Cancer 2017;79:50-60.

12. Geissler M, Riera-Knorrenschild J, Martens UM, et al. 
Final results and OS of the randomized phase II VOLFI trial (AIO- KRK0109): mFOLFOXIRI + panitumumab versus FOLFOXIRI as first-line treatment in patients with RAS wild- type metastatic colorectal cancer (mCRC). J Clin Oncol 2019;37:3511.

13. Le DT, Uram JN, Wang H, et al. PD-1 Blockade in Tumors with Mismatch-Repair Deficiency. N Engl J Med 2015;372:2509-20.

14. Le DT, Durham JN, Smith KN, et al. Mismatch repair deficiency predicts response of solid tumors to PD-1 blockade. Science 2017;357:409-13.

15. Overman MJ, Lonardi S, Wong KYM, et al. Durable Clinical Benefit With Nivolumab Plus Ipilimumab in DNA Mismatch Repair-Deficient/Microsatellite Instability-High Metastatic Colorectal Cancer. J Clin Oncol 2018;36:773-9.

16. Overman MJ, McDermott R, Leach JL, et al. Nivolumab in patients with metastatic DNA mismatch repair-deficient or microsatellite instability-high colorectal cancer (CheckMate 142): an open-label, multicentre, phase 2 study. Lancet Oncol 2017;18:1182-91.

17. Yaeger R, Cercek A, Chou JF, et al. BRAF mutation predicts for poor outcomes after metastasectomy in patients with metastatic colorectal cancer. Cancer 2014;120:2316-24.

18. Gagnière J, Dupré A, Gholami SS, et al. Is Hepatectomy Justified for BRAF Mutant Colorectal Liver Metastases?: A Multi-institutional Analysis of 1497 Patients. Ann Surg 2018. [Epub ahead of print].

19. Kopetz S, Desai J, Chan E, et al. Phase II Pilot Study of

Cite this article as: de la Fouchardiere C. Treating BRAFV600E metastatic colorectal patients in 2019: a BEACON of hope? Ann Transl Med 2019;7(21):601. doi: 10.21037/atm.2019.08.110
Vemurafenib in Patients With Metastatic BRAF-Mutated Colorectal Cancer. J Clin Oncol 2015;33:4032-8.

20. Corcoran RB, Atreya CE, Falchook GS, et al. Combined BRAF and MEK Inhibition With Dabrafenib and Trametinib in BRAF V600-Mutant Colorectal Cancer. J Clin Oncol 2015;33:4023-31.

21. van Geel RM, Tabernero J, Elez E, et al. A Phase Ib DoseEscalation Study of Encorafenib and Cetuximab with or without Alpelisib in Metastatic BRAF-Mutant Colorectal Cancer. Cancer Discov 2017;7:610-9.

22. Kopetz S, McDonough SL, Lenz HJ, et al. Randomized trial of irinotecan and cetuximab with or without vemurafenib in BRAF-mutant metastatic colorectal cancer (SWOG S1406). J Clin Oncol 2017;35:3505.

23. Van Cutsem E, Cuyle PJ, Huijberts S, et al. BEACON CRC study safety lead-in (SLI) in patients with BRAFV600E metastatic colorectal cancer (mCRC): Efficacy and tumor markers. J Clin Oncol 2018;36:627.

24. Colloca GA, Venturino A, Guarneri D. Early tumor shrinkage after first-line medical treatment of metastatic colorectal cancer: a meta-analysis. Int J Clin Oncol 2019;24:231-40.

25. Kopetz S, Grothey A, Van Cutsem E, et al. LBA006BEACON CRC: a randomized, 3-Arm, phase 3 study of encorafenib and cetuximab with or without binimetinib vs. choice of either irinotecan or FOLFIRI plus cetuximab in BRAF V600E-mutant metastatic colorectal cancer. Ann Oncol 2019;30:mdz183.004. 